Gefässchirurgie 2016 · 21:613-614

DOI 10.1007/s00772-016-0219-6

Online publiziert: 8. November 2016

C) Springer Medizin Verlag Berlin 2016

CrossMark

\section{Erratum zu:}

Gefässchirurgie (2016) 21:418-425

doi:10.1007/s00772-016-0192-0

In diesem Beitrag wurde die deutsche Zusammenfassung unvollständig wiedergegeben. Die vollständige Zusammenfassung finden Sie anbei.

Der Verlag bittet, den Fehler zu entschuldigen.

\section{Korrespondenzadresse}

\section{Prof. Dr. D. Böckler}

Klinik für Gefäßchirurgie und Endovaskuläre Chirurgie, Universitätsklinikum Heidelberg Im Neuenheimer Feld 110, 69120 Heidelberg, Deutschland

dittmar.boeckler@med.uni-heidelberg.de

D. Böckler' $\cdot$ K. Meisenbacher ${ }^{1}$ A. S. Peters ${ }^{1} \cdot$ C. Grond-Ginsbach $^{2}$ - M. S. Bischoff

${ }^{1}$ Klinik für Gefäßchirurgie und Endovaskuläre Chirurgie, Universitätsklinikum Heidelberg, Heidelberg, Deutschland

${ }^{2}$ Klinik für Neurologie, Universitätsklinikum Heidelberg, Heidelberg, Deutschland

\title{
Erratum zu: Endovaskuläre Therapie genetisch bedingter Aortenerkrankungen
}

\section{Zusammenfassung $\cdot$ Abstract}

Gefässchirurgie DOI 10.1007/s00772-016-0192-0

(c) Springer Medizin Verlag Berlin 2016

\section{Böckler · K. Meisenbacher · A. S. Peters · C. Grond-Ginsbach · M. S. Bischof \\ Endovaskuläre Therapie genetisch bedingter Aortenerkrankungen}

Zusammenfassung

Hintergrund. Die wichtigsten Strukturproteine der Gefäßwand sind Kollagen und Elastin. Genetisch bedingte Bindegewebserkrankungen führen zu Degeneration und Aneurysmabildung, spontanen Dissektionen oder Rupturen von Arterien. Am bekanntesten sind das Marfan-Syndrom, das Ehlers-Danlos-Syndrom vom vaskulären Typ, das Loeys-Dietz-Syndrom sowie familiäre Aortenaneurysmen und Dissektionen.

Fragestellung. Welchen Stellenwert haben endovaskuläre Behandlungsoptionen in der Therapie aortaler Pathologien auf dem Boden von Bindegewebserkrankungen?

Material und Methoden. Auswertung aktueller randomisierter Untersuchungen und Registerstudien.

Ergebnis. Die Therapie der Wahl der meist bereits in jungem Alter betroffenen Patienten ist primär konservativ bzw. offen-chirurgisch. Zur endovaskulären Therapie abdomineller Aneurysmen (EVAR) oder thorakalen Aortenpathologien (TEVAR) gibt es nur wenig Evidenz.

Schlussfolgerungen. Die Progression der genetisch bedingten Grunderkrankung mit konsekutiver Aortendilatation führt zu sekundären Endoleckagen und vermehrten Reinterventionen mit unsicherem Langzeitergebnis. Deshalb besteht derzeit Konsensus, dass EVAR und TEVAR bei genetisch bedingten Aortenerkrankungen auf begründete Ausnahmefälle und Notfallsituationen begrenzt sein sollten.

Schlüsselwörter Bindegewebserkrankung · Marfan · Aorta . EVAR $\cdot$ TEVAR 
Hier steht eine Anzeige.

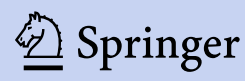

